\title{
Phase Ib Study of Osimertinib Plus Ramucirumab in Japanese Lung Cancer Patients With EGFR Mutation
}

\author{
HIROAKI AKAMATSU ${ }^{1}$, YUICHI OZAWA ${ }^{1}$, JUN OYANAGI ${ }^{1}$, DAICHI FUJIMOTO ${ }^{2}$, AKITO HATA $^{3}$, \\ NOBUYUKI KATAKAMI ${ }^{2,4}$, KEISUKE TOMII ${ }^{2}$, ERIKO MURAKAMI ${ }^{1}$, TAKEYA SUGIMOTO ${ }^{1}$, \\ TOSHIO SHIMOKAWA ${ }^{5}$, YASUHIRO $\mathrm{KOH}^{1}$ and NOBUYUKI YAMAMOTO ${ }^{1}$ \\ ${ }^{1}$ Internal Medicine III, Wakayama Medical University, Wakayama, Japan; \\ ${ }^{2}$ Department of Respiratory Medicine, Kobe City Medical Center General Hospital, Kobe, Japan; \\ ${ }^{3}$ Department of Thoracic Oncology, Kobe Minimally Invasive Cancer Center, Kobe, Japan; \\ ${ }^{4}$ Department of Medical Oncology, Takarazuka City Hospital, Takarazuka, Japan; \\ ${ }^{5}$ Clinical Study Support Center, Wakayama Medical University, Wakayama, Japan
}

\begin{abstract}
Background/Aim: To explore the safety of osimertinib plus ramucirumab in patients with EGFR-mutated lung adenocarcinoma. Patients and Methods: Six advanced lung adenocarcinoma patients with EGFR mutation were treated with osimertinib $80 \mathrm{mg} /$ day plus ramucirumab $10 \mathrm{mg} / \mathrm{kg}$, every two weeks. Defined dose-limiting toxicity (DLT) was assessed within the first two treatment cycles. Results: Of those enrolled, five patients had both EGFR exon 20 T790M mutation and sensitizing mutation. DLT was observed in one patient (grade 3 appetite loss). During the entire period, no other severe adverse event was observed. Five patients showed partial response and one disease progression. Median progression-free survival for patients with EGFR T790M was 9.2 months. In an exploratory analysis, changes of cell-free DNA at 2 weeks predicted radiological tumor responses. Conclusion: The safety results of osimertinib plus ramucirumab in Japanese lung adenocarcinoma patients with EGFR mutation will lead to further efficacy investigation.
\end{abstract}

Epidermal growth factor receptor (EGFR) mutation is known as an oncogenic driver in advanced lung adenocarcinoma and

This article is freely accessible online.

This study was presented at the Japanese Lung Cancer Society Annual Meeting at Osaka, Dec 2019.

Correspondence to: Hiroaki Akamatsu, MD, Ph.D., Internal Medicine III, Wakayama Medical University, 811-1, Kimiidera, Wakayama, 641-8509 Japan. Tel: +81 734410619, Fax: +81 734462877, e-mail: hiroakiakamatsu@gmail.com

Key Words: Osimertinib, ramucirumab and epidermal growth factor receptor $(E G F R)$ mutation, liquid biopsy. its frequency has been reported to be approximately $40 \%$ in East Asians (1). Until 2018, first- or second-generation EGFR-tyrosine kinase inhibitors (TKIs) were the standard treatment for lung adenocarcinoma patients with EGFR mutation. They allowed almost one year of progression-free survival (PFS), but ultimately developed resistance. Of those progressed, EGFR T790M mutation is the most common resistance mechanism (2).

Osimertinib, the first-in class third-generation EGFR-TKI, has a broad spectrum of activity in various types of EGFRmutated cell lines including those carrying the EGFR T790M mutation (3). Its inhibitory effect on EGFR wild-type is minute, so clinical toxicities are mild. AURA3 was the first phase III study to compare osimertinib with platinum doublet chemotherapy in EGFR-mutated patients that progressed with EGFR-TKIs and acquired EGFR T790M mutation (4). Another phase 3 trial, FLAURA, compared osimertinib with first generation EGFR-TKIs among treatment-naïve patients harboring sensitizing EGFR mutations (5). Osimertinib was shown to prolong PFS in both studies. Based on these, current clinical guidelines suggest osimertinib as the first choice in both situations.

Vascular endothelial growth factor (VEGF) plays various roles in cancer evolution, including angiogenesis, proliferation and metastasis. In EGFR-mutated lung cancer, a preclinical study suggested that inhibition of VEGF may work synergistically with EGFR inhibitors (6). Ramucirumab is an anti-VEGF receptor2 antibody that has already been approved for the treatment of various malignancies. In EGFR-mutated non-small cell lung cancer (NSCLC), erlotinib plus ramucirumab has been tested and significant prolongation of PFS has been shown in a phase III trial (7). These clinical results prompted us to explore the combination of osimertinib with ramucirumab, and to clarify the safety profile in Japanese patients. 


\section{Patients and Methods}

Study design. This is an open-label, single arm phase Ib study to explore the safety of osimertinib plus ramucirumab in Japanese lung adenocarcinoma patients with $E G F R$ mutation. In previous studies, osimertinib monotherapy had a less-toxic profile compared with first-generation EGFR-TKIs. Moreover, the combination of erlotinib with anti-VEGF antibodies was tolerable in phase 3 trials. Although conventional phase I studies usually set dose escalation cohorts with a $3+3$ design, this evidence allowed us to test this combination with full doses in six patients.

The primary endpoint of this study was safety during the first two cycles. Secondary endpoints are overall response rate (ORR), progression-free survival (PFS), overall survival (OS), and safety during the entire period. Dose-limiting toxicity (DLT) was defined as any of the following: hemoglobin decrease (grade 4), platelet decrease ( $\geq$ grade 3 ), neutropenia (grade 4 , lasting $\geq$ eight days), febrile neutropenia, skin rash (grade 4 ), proteinuria ( $\geq 3 \mathrm{~g} /$ day, 2 $\mathrm{g}$ /day that lasts $\geq 3$ times or does not recover to $\leq 2 \mathrm{~g}$ /day within 2 weeks), hypertension (grade 4), or other non-hematological toxicities except for electrolyte abnormalities and hyperglycemia $(\geq$ grade 3). If 2 or more of the six patients had DLT, this combination would be determined as not tolerable and the study would be terminated.

Eligibility and exclusion criteria. Eligible participants were those with pathologically proven lung adenocarcinoma with clinical stage IIIB, IV, or recurrence after curative surgery or radiotherapy. Patients without prior history of EGFR-TKI should harbor EGFR sensitizing mutation (exon 19 deletion, or exon 21 L858R). If patients had progression with prior EGFR-TKI other than osimertinib, they should be confirmed to have EGFR T790M. EGFR mutation testing was performed using tumor specimen or cell-free DNA extracted from peripheral blood at local facilities by approved PCR-based methods. Other inclusion criteria were age $\geq 20$ years, ECOG performance status (PS) of 0-1, measurable lesion, and adequate organ function within 14 days prior to registration. Written informed consented was obtained from all patients.

Patients were excluded if they met any of the following criteria: history of interstitial pneumonia or evidence of interstitial lung disease by computed tomography (CT) scan, inability to swallow or ingest, considered to be at high risk of bleeding (i.e. history of hemoptysis or gastrointestinal bleeding), history of embolisms in the three months prior to protocol therapy, serious illness or medical condition (i.e. symptomatic or recent cerebrovascular disease, uncontrolled pleural effusion, or uncontrolled hypertension), localized infection requiring procedure or active systemic infection, positivity for hepatitis B virus antigen, leptomeningeal carcinomatosis, other malignancies, pregnant or nursing women, women unwilling to use adequate contraceptive methods, serious psychiatric condition making registration difficult, or serious known allergy. Patients with brain metastasis were allowed if he/she was asymptomatic, but those who had leptomeningeal carcinomatosis were excluded.

Study treatment and assessment procedures. Treatment consisted of osimertinib plus ramucirumab. Beginning on day $1,80 \mathrm{mg}$ of osimertinib was administered every day, until disease progression. Also beginning on day $1,10 \mathrm{mg} / \mathrm{kg}$ of ramucirumab was administered every two weeks, until disease progression.
To assess efficacy, patients underwent chest CT every eight weeks until 72 weeks, and every 12 weeks thereafter. Gadoliniumenhanced MRI of the brain was performed at baseline prior to registration for all patients. Adverse events (AEs) were graded using Common Terminology Criteria for Adverse Events, version 4.0.

Biomarker analysis. Before and after treatment, $20 \mathrm{ml}$ of peripheral blood (at two, four, eight and 16 weeks) were collected and centrifuged to separate plasma. The collected plasma was stored at $-80^{\circ} \mathrm{C}$ until cell-free DNA (cfDNA) purification. After thawing, the plasma was centrifuged at $1500 \times g$ for $10 \mathrm{~min}$ at $4^{\circ} \mathrm{C}$ and the supernatant was used for cfDNA purification. cfDNA was purified with QIAamp circulating nucleic acid kit (QIAGEN, Hilden, Germany) according to the manufacturer's instructions. The purified cfDNA was quantified with a Qubit 2.0 fluorometer using Qubit dsDNA HS assay kit (Thermo Fisher Scientific, Waltham, MA, USA). To assess the heterogeneity of the tumor, genetic alterations in cfDNA were evaluated by next generation sequencing (NGS) [AVENIO ctDNA surveillance kit (Roche Diagnostics, Pleasanton, CA, USA)]. According to the manufacturer's instructions, sequencing libraries were prepared, and the quality was evaluated by Qubit assay and High Sensitivity D5000 ScreenTape on a TapeStation 2200 (Agilent, Santa Clara, CA, USA). After the quality control, the libraries were pooled and sequenced on an Illumina Nextseq 500 using 300 cycles high output kit v2.5 (Illumina, San Diego, CA, USA). After sequencing, genetic alterations were analyzed using AVENIO oncology analysis software (Roche Sequencing Solutions, Inc., Pleasanton, CA, USA). To confirm the results obtained by NGS, droplet digital PCR (ddPCR) was performed for three common types of EGFR mutation (exon 19 deletion, exon $20 \mathrm{~T} 790 \mathrm{M}$ and exon $21 \mathrm{~L} 858 \mathrm{R}$ ) using RainDrop digital PCR system (Bio-Rad Laboratories, Hercules, CA, USA) $(8,9)$.

Statistical analysis. For the ORR, the point estimates and the $95 \%$ confidence interval (CI) are provided. PFS was estimated using the Kaplan-Meier (KM) curves. Using KM method, median PFS and PFS rate at one-year were also analyzed with 95\%CI. OS was analyzed similar to PFS and median OS and OS rate at one-year were also analyzed. Statistical analyses were conducted with JMP software (version 11, SAS Institute, Cary, NC, USA) and GraphPad Prism ver.7.00 for Windows (GraphPad Software, San Diego, CA, USA).

Ethical considerations. The study was conducted in compliance with the principles of the Declaration of Helsinki, and the protocol was approved by the institutional review board of each participating institution. Written informed consent was obtained from all patients before any screening or inclusion procedures. This protocol was registered in the University Hospital Medical Information Network, Japan (protocol identification no. UMIN000023761 and UMIN000030164).

\section{Results}

Patient characteristics. Baseline characteristics of the six patients are shown in Table I. Median age was 67 (range=5571 years), four were male and five had ECOG PS of 1. One patient did not have history of EGFR-TKI treatment and five progressed following prior treatment with EGFR-TKIs and 
Table I. Baseline characteristics.

\begin{tabular}{lc}
\hline Characteristics & $\mathrm{N}=6$ \\
\hline Age; median (range) & $67(55-71)$ \\
Gender; male/female & $4 / 2$ \\
C-stage; III-IV/post-operative recurrence & $5 / 1$ \\
Site of EGFR mutation & \\
Exon19del & 3 \\
Exon 20 T790M & 5 \\
Exon 21 L858R & 3 \\
No. of prior treatment; 0/1- & $1 / 5$ \\
ECOG Performance status; 0/1 & $4 / 2$ \\
Smoking status; non or light smoker/smoker & $3 / 3$ \\
\hline
\end{tabular}

c-stage: Clinical stage; EGFR: epidermal growth factor receptor and ECOG: Eastern Cooperative Oncology Group.

Table II. Adverse events during two cycles (primary endpoint).

\begin{tabular}{llll}
\hline & G1 & G2 & $\geq \mathrm{G} 3$ \\
\hline Platelet decrease & 4 & 0 & 0 \\
Diarrhea & 3 & 0 & 0 \\
Skin rash & 3 & 0 & 0 \\
Proteinuria & 1 & 1 & 0 \\
Appetite loss & 0 & 0 & $1^{*}$ \\
Vomiting & 0 & 1 & 0 \\
Hypertension & 0 & 1 & 0 \\
AST elevation & 1 & 0 & 0 \\
ALT elevation & 1 & 0 & 0 \\
Liver dysfunction & 1 & 0 & 0 \\
Nausea & 1 & 0 & 0 \\
Rash maculopapular & 1 & 0 & 0 \\
Nasal bleeding & 1 & 0 & 0 \\
\hline
\end{tabular}

*Dose-limiting toxicity. AST: Aspartate transaminase; ALT: alanine amino transferase.

acquired EGFR T790M mutation. At the data cut-off, median follow-up was 18.7 months (range=12.6-25.1 months). One patient withdrew consent after 89 days of treatment, so we could not acquire further information from this patient.

Safety and drug exposure. Table II shows the toxicity profile during the DLT assessment period. Although one DLT occurred (grade 3 appetite loss), other AEs were $\leq$ grade 2 . One patient developed grade 3 appetite loss on day 22 that required admission. She received peripheral intravenous fluids and recovered within one month.

Table III summarizes AEs during the entire period. Similar to those during the DLT assessment period, there were no severe AEs other than DLT. Other common AEs were platelet decrease, diarrhea $(n=4$ each), skin rash, and hypertension $(n=3$ each). One patient had grade 2 interstitial lung disease
Table III. Adverse events during entire period.

\begin{tabular}{llll}
\hline & G1 & G2 & $\geq \mathrm{G} 3$ \\
\hline Platelet decrease & 4 & 0 & 0 \\
Diarrhea & 3 & 1 & 0 \\
Hypertension & 1 & 2 & 0 \\
Skin rash & 3 & 0 & 0 \\
Proteinuria & 1 & 1 & 0 \\
ALT elevation & 2 & 0 & 0 \\
Nasal bleeding & 2 & 0 & 0 \\
Appetite loss & 0 & 0 & 1 \\
WBC decrease & 0 & 1 & 0 \\
Neutropenia & 0 & 1 & 0 \\
Vomiting & 0 & 1 & 0 \\
Mucositis oral & 0 & 1 & 0 \\
Interstitial lung disease & 0 & 1 & 0 \\
Hb decrease & 1 & 0 & 0 \\
AST elevation & 1 & 0 & 0 \\
Liver dysfunction & 1 & 0 & 0 \\
Nausea & 1 & 0 & 0 \\
Dry skin & 1 & 0 & 0 \\
Rash maculopapular & 1 & 0 & 0 \\
Paronychia & 1 & 0 & 0 \\
\hline
\end{tabular}

AST: Aspartate transaminase; ALT: alanine aminotransferase; Hb: hemoglobin; WBC: white blood cell.

on day 36 , but finally recovered on day 62 . At the time of analysis, median exposure of osimertinib administration was $100 \%$ of the planned dosage (range $=76-100 \%$ ) and median cycles of ramucirumab administration was five (range=2-9).

Efficacy and post-study therapy. Five patients had partial response and only one had progressive disease (PD) as best response. Thus, ORR was $83 \%(95 \% \mathrm{CI}=42-99 \%)$. Swimmer's plot of all patients and Kaplan-Meier curve of PFS among patients with EGFR T790M are shown in Figure 1. Among patients with T790M, median PFS was 9.2 months and oneyear PFS was $38 \%(95 \% \mathrm{CI}=36-43 \%)$. No patients died at the time of analysis, so survival rate at one-year was $100 \%$ and median overall survival was not reached (data not shown).

At the time of analysis, five discontinued study treatment (one due to DLT and four due to PD). Of those, three received osimertinib monotherapy as their post-therapy (Figure 1a). Patient no.1, who had DLT, received $40 \mathrm{mg}$ of osimertinib monotherapy and is still maintaining her disease for 2 years. Patient no. 3 had clinical progression (worsening pain due to bone metastasis) on day 112 . This patient resumed osimertinib monotherapy at the same dose after palliative radiotherapy and this has been effective for 19 months. Patient no.6 discontinued study treatment due to interstitial lung disease. After platinum doublet chemotherapy, he received osimertinib re-challenge as the third-line therapy and it worked for over eight months. 

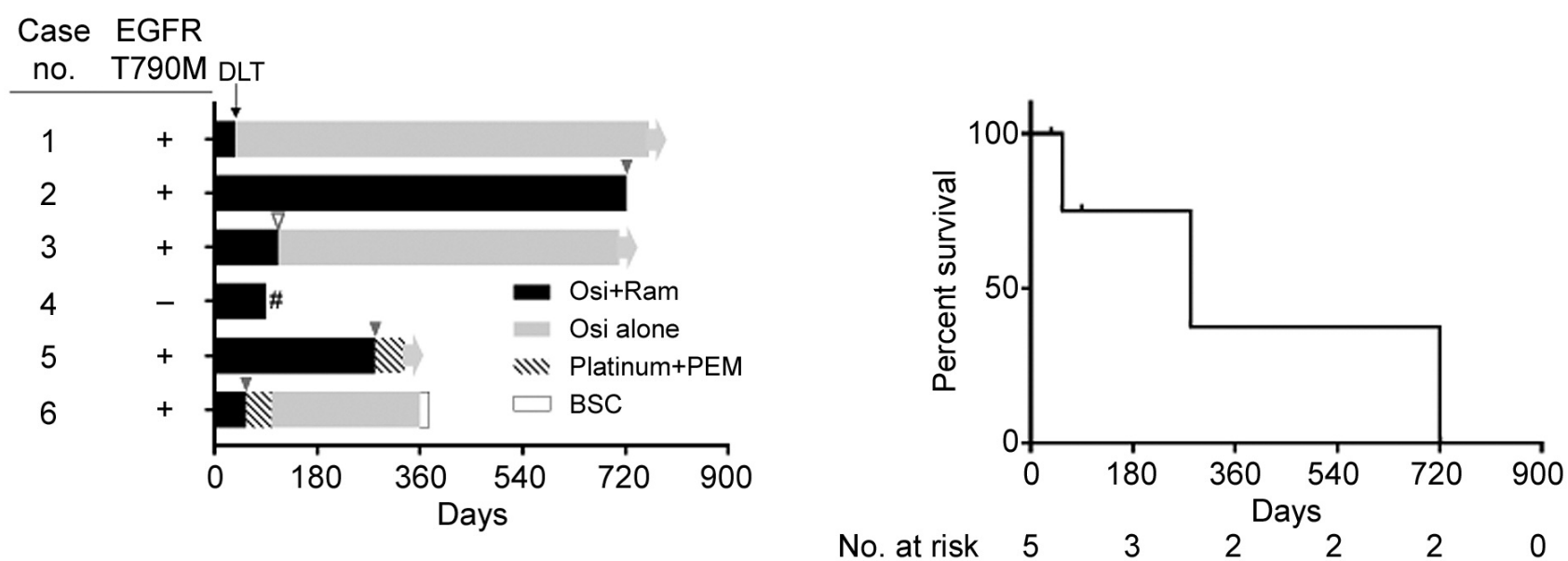

Figure 1. Efficacy of osimertinib plus ramucirumab. a) Swimmer's plot of all participants and b) Kaplan-Meier curves of progression-free survival in patients with EGFR T790M mutation. Triangles, triangle outlined with black and \# indicate systemic disease progression, local progression and withdrawn from the trial, respectively. DLT: Dose-limiting toxicity; Osi: osimertinib; Ram: ramucirumab; PEM: pemetrexed; BSC: best supportive care.

Table IV. Detected gene alterations at baseline.

\begin{tabular}{|c|c|c|c|c|c|}
\hline \multirow[t]{2}{*}{ Patient no } & \multicolumn{2}{|c|}{ Digital PCR } & \multicolumn{3}{|c|}{ Next generation sequencing (AVENIO) } \\
\hline & Site of EGFR mutations & Allele frequency $(\%)$ & Site of EGFR mutations & Allele frequency $(\%)$ & $\mathrm{CNV} /$ fusion \\
\hline \multirow[t]{2}{*}{1} & Exon 19 del & 6.2 & Exon 19 del & 3.9 & EGFR amp \\
\hline & Exon $20 \mathrm{~T} 790 \mathrm{M}$ & 2.6 & Exon $20 \mathrm{~T} 790 \mathrm{M}$ & 3.2 & \\
\hline \multirow[t]{5}{*}{2} & Exon 19 del & 1.5 & Exon $19 \mathrm{del}$ & & EGFR amp \\
\hline & Exon $20 \mathrm{~T} 790 \mathrm{M}$ & 17.2 & p.Glu746_Arg748del & 1.8 & \\
\hline & & & p.Glu746_Arg750del & 0.2 & \\
\hline & Exon 21 L858R & 0.09 & Exon $20 \mathrm{~T} 790 \mathrm{M}$ & 1.6 & \\
\hline & & & Exon $20 \mathrm{C} 797 \mathrm{~S}$ & 0.7 & \\
\hline \multirow[t]{2}{*}{3} & Exon 19 del & 11.1 & Exon 19 del & 7.4 & \\
\hline & Exon $20 \mathrm{~T} 790 \mathrm{M}$ & 4.2 & Exon $20 \mathrm{~T} 790 \mathrm{M}$ & 5.5 & \\
\hline
\end{tabular}

Biomarker analysis. Of six participants, three consented to participate in biomarker analysis. Table IV shows gene alterations detected by ddPCR or NGS at baseline. All EGFR mutations detected by ddPCR were also captured by NGS. In addition, we could detect other EGFR mutations like exon 20 C797S in patient no.2, and EGFR amplifications in patients no. 1 and 2. Figure 2 shows longitudinal monitoring by RECIST or allele frequency (AF) of EGFR T790M in cfDNA. All patients showed rapid decrease of cfDNA within two weeks and this reflected radiological tumor shrinkage at 8 weeks. Interestingly, patient no.3 was judged to have PD due to worsening pain because of bone metastasis, but AF was not changed at this timepoint. This patient successfully resumed osimertinib monotherapy after palliative radiotherapy.

\section{Discussion}

EGFR-TKI plus anti-VEGF inhibitor is one of the most promising combination strategies in EGFR-mutated NSCLC. Although two phase III trials with erlotinib plus either bevacizumab or ramucirumab demonstrated significant prolongation of PFS (10), there is limited data on osimertinib plus anti-VEGF agents. This is therefore the first study to show the tolerability of osimertinib plus ramucirumab in Japanese lung adenocarcinoma patients. The number of participants was small, but AE profiles were generally mild. We had one case of severe AE (appetite loss), but the patient fully recovered.

Among five patients with EGFR T790M mutation, median PFS was unremarkable. Until now, several studies have 


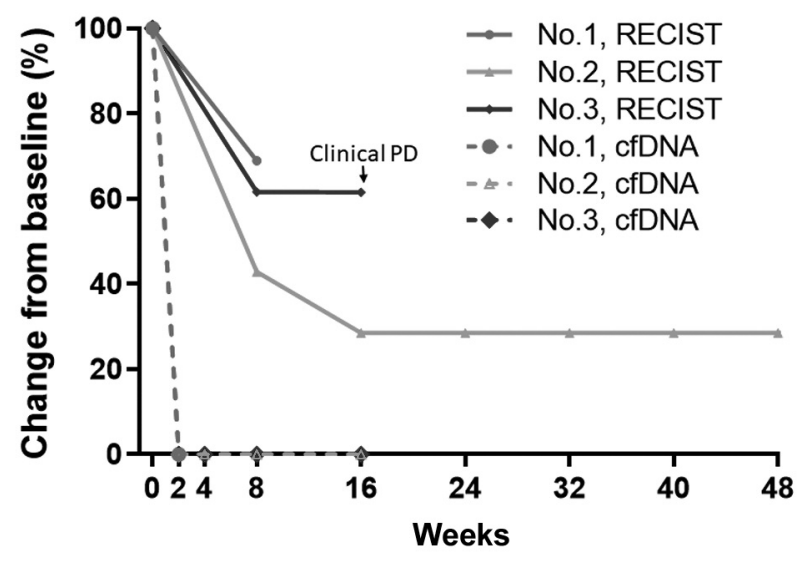

Figure 2. Longitudinal assessment by RECIST and liquid biopsy. Regarding cfDNA, relative changes in allele frequency of EGFR T790M from baseline were plotted. PD: Progressive disease.

reported preliminary results of osimertinib in combination with anti-VEGF antibodies; median PFS was 18.4 months in the first-line and 11.0 months in the later line in patients with EGFR T790M $(11,12)$. Recently, a Japanese randomized phase II study examining osimertinib with or without bevacizumab in T790M-mutated patients failed to show prolongation of PFS (13). A phase II study already completed accrual in Japan (UMIN 000030206) and another phase III trial in the U.S. is ongoing (NCT04181060). These results are eagerly anticipated.

Our biomarker analysis revealed the utility of tracking the change of cfDNA in predicting radiological response, which is consistent with prior studies (14). Although most patients respond to EGFR-TKI, it is still useful to develop lessinvasive methods like ours to detect intrinsic resistance early. On the other hand, whether plasma progression is more sensitive and clinically meaningful than RECIST has been debated. In our study, patient no. 3 did not show significant increase of $\mathrm{AF}$ at the time of local progression. Conversely, patient no. 2 showed an increase in AF at 8 weeks without any manifestation of progression. Such dynamics unrelated to disease control have been shown in a previous report, but their clinical implication has not been clarified (15). Significance of plasma progression compared with radiological progression will be explored in larger prospective trials. Using highly sensitive NGS, we could detect the same EGFR mutations detected by ddPCR. In addition, several gene alterations, such as EGFR C797S or $E G F R$ gene amplification which are known as resistant to osimertinib, were detected by NGS (16). Although the clinical relevance was unclear due to small sample size, our results suggest the applicability of NGS. Especially, detecting EGFR mutations such as C797S that makes the tumor sensitive to first- or second-generation EGFR-TKIs may help to consider subsequent therapy (17).

In conclusion, the safety results of osimertinib plus ramucirumab in Japanese lung adenocarcinoma patients with $E G F R$ mutation will lead to further efficacy investigation.

\section{Conflicts of Interest}

Hiroaki Akamatsu received honoraria from AstraZeneca K.K., Boehringer Ingelheim Japan Inc., Bristol-Myers Squibb, Chugai Pharmaceutical Co. Ltd., Eli Lilly Japan K.K., MSD K.K., Novartis Pharma K.K., Ono Pharmaceutical Co. Ltd. and Taiho Pharmaceutical Co. Ltd. He received research funding from Chugai Pharmaceutical Co. Ltd., MSD K.K. Yuichi Ozawa received honoraria from AstraZeneca K.K., Chugai Pharmaceutical Co. Ltd., Eli Lilly Japan K.K., MSD K.K., Ono Pharmaceutical Co. Ltd. and Taiho Pharmaceutical Co. Ltd. He had an advisory role for AstraZeneca K.K. Daichi Fujimoto received honoraria from AstraZeneca K.K., Boehringer Ingelheim Japan Inc., Bristol-Myers Squibb, Chugai Pharmaceutical Co. Ltd., Eli Lilly Japan K.K., MSD K.K., Novartis Pharma K.K., Ono Pharmaceutical Co. Ltd. and Taiho Pharmaceutical Co. Ltd. He received research funding from AstraZeneca K.K. Akito Hata received honoraria from AstraZeneca K.K., Boehringer Ingelheim Japan Inc., Chugai Pharmaceutical Co. Ltd., and Eli Lilly Japan K.K. He received research funding from AstraZeneca K.K., Boehringer Ingelheim Japan Inc., Eli Lilly Japan K.K., and MSD. Nobuyuki Katakami received honoraria from AstraZeneca K.K., Boehringer Ingelheim Japan Inc., Bristol-Myers Squibb, Chugai Pharmaceutical Co. Ltd., Eli Lilly Japan K.K., MSD K.K., Novartis Pharma K.K., Ono Pharmaceutical Co. Ltd., Taiho Pharmaceutical Co. Ltd. and Japan Pharmaceutical Association. Keisuke Tomii received honoraria from Astellas Pharma Inc., AstraZeneca K.K., Boehringer Ingelheim Japan Inc., Chugai Pharmaceutical Co. Ltd., Daiichi Sankyo, Eli Lilly Japan K.K., GlaxoSmithKline Pharmaceuticals Ltd., Kyorin Pharmaceutical co. Ltd, Kyowa Hakko Kirin Co., Ltd, MSD K.K., Novartis Pharma K.K, Sanofi K.K., Shionogi \& Co., Ltd., Taiho Pharmaceutical Co. Ltd. and Teijin Pharma, Ltd. Takeya Sugimoto received honoraria from AstraZeneca K.K. Yasuhiro Koh received honoraria from AstraZeneca K.K., Boehringer Ingelheim Japan Inc, Bristol-Myers Squibb, Chugai Pharmaceutical Co. Ltd., Eli Lilly Japan K.K., Ono Pharmaceutical Co. Ltd. and Thermo Fisher, Tosoh Corp. He also received research funding from AstraZeneca K.K., Boehringer Ingelheim Japan Inc, Bristol-Myers Squibb, Chugai Pharmaceutical Co. Ltd., MSD, On-chip Biotechnologies, Ono Pharmaceutical Co. Ltd, Terumo and Toppan Printing. Nobuyuki Yamamoto received honoraria from AstraZeneca K.K., Boehringer Ingelheim Japan Inc., Bristol-Myers Squibb, Chugai Pharmaceutical Co. Ltd., Daiichi Sankyo, Eli Lilly Japan K.K., Life Technologies, Merck Biopharma Co., Ltd., MSD K.K., Nippon Kayaku Co., Ltd., Novartis Pharma K.K., Ono Pharmaceutical Co. Ltd., Pfizer Japan Inc., Takeda Pharmaceutical, Taiho Pharmaceutical Co. Ltd. and Thermo Fisher Scientific K.K. He received research funding from Abbvie GK, Amgen, AstraZeneca K.K., Astellas Pharma Inc, Boehringer Ingelheim Japan Inc., Chugai Pharmaceutical Co. Ltd., Daiichi Sankyo, Eisai Co., Ltd., Eli Lilly Japan K.K., Kyorin Pharmaceutical co. Ltd, MSD K.K., Novartis Pharma K.K., Ono Pharmaceutical Co. Ltd., Pfizer Japan Inc., Takeda Pharmaceutical, Taiho Pharmaceutical Co. Ltd., Terumo corp., TOPPAN PRINTING 
Co., Ltd., TOSO Co, Ltd. and Tsumura \& Co. Jun Oyanagi, Eriko Murakami and Toshio Shimokawa have no financial relationships to disclose.

\section{Authors' Contributions}

All Authors had full access to the data of this analysis and take responsibility for all the content of the manuscript. Study concept and design: Akamatsu, Koh and Yamamoto. Accrual of the participants: Akamatsu, Ozawa, Fujimoto, Hata, Katakami, Tomii, Murakami and Sugimoto. Acquisition, analysis, or interpretation of data: Akamatsu, Oyanagi, Koh and Yamamoto. Drafting of the manuscript: Akamatsu, Oyanagi, Koh and Yamamoto. Statistical analysis: Shimokawa. Study supervision: Akamatsu and Yamamoto.

\section{Acknowledgements}

The Authors are grateful to data managers and other support staff (Especially, Ms. Yoko Yabuuchi) at the Wakayama Medical University Data Center. The Authors acknowledge proofreading and editing by Benjamin Phillis at the Clinical Study Support Center, Wakayama Medical University.

\section{Funding}

This work was supported by Eli Lilly.

\section{References}

1 Dearden S, Stevens J, Wu YL and Blowers D: Mutation incidence and coincidence in non-small-cell lung cancer: meta-analyses by ethnicity and histology (mutMap). Ann Oncol 24(9): 2371-2376, 2013. PMID: 23723294. DOI: 10.1093/annonc/mdt205

2 Arcila ME, Oxnard GR, Nafa K, Riely GJ, Solomon SB, Zakowski MF, Kris MG, Pao W, Miller VA and Ladanyi M: Rebiopsy of lung cancer patients with acquired resistance to EGFR inhibitors and enhanced detection of the T790M mutation using a locked nucleic acid-based assay. Clin Cancer Res 17(5): 1169-1180, 2011. PMID: 21248300. DOI: 10.1158/1078-0432.CCR-10-2277

3 Cross DA, Ashton SE, Ghiorghiu S, Eberlein C, Nebhan CA, Spitzler PJ, Orme JP, Finlay MRV, Ward RA, Mellor MJ, Hughes G, Rahi A, Jacobs VN, Brewer MR, Ichihara E, Sun J, Jin H, Ballard P, Al-Kadhimi K, Rowlinson R, Klinowska T, Richmond GHP, Cantarini M, Kim DW, Ranson MR and Pao W: AZD9291, an irreversible EGFR TKI, overcomes T790M-mediated resistance to EGFR inhibitors in lung cancer. Cancer Discov 4(9): 1046-1061, 2014. PMID: 24893891. DOI: 10.1158/2159-8290.CD-14-0337

4 Mok TS, Wu Y-L, Ahn M-J, Garassino MC, Kim HR, Ramalingam SS, Shepherd FA, He Y, Akamatsu H, Theelen WS, Lee CK, Sebastian M, Templeton A, Mann H, Marotti M, Ghiorghiu S, Papadimitrakopoulou VA and AURA3 Investigators. Osimertinib or platinum-pemetrexed in EGFR T790M-positive lung cancer. N Engl J Med 376(7): 629-640, 2017. PMID: 27959700. DOI: 10.1056/NEJMoa1612674

5 Soria JC, Ohe Y, Vansteenkiste J, Reungwetwattana T, Chewaskulyong B, Lee KH, Dechaphunkul A, Imamura F, Nogami N, Kurata T, Okamoto I, Zhou C, Cho BC, Cheng Y, Cho EK, Voon PJ, Planchard D, Su WC, Gray JE, Lee SM,
Hodge R, Marotti M, Rukazenkov Y, Ramalingam SS and FLAURA Investigators: Osimertinib in untreated EGFR-mutated advanced non-small-cell lung cancer. N Engl J Med 378(2): 113125, 2018. PMID: 29151359. DOI: 10.1056/NEJMoa1713137

6 Naumov GN, Nilsson MB, Cascone T, Briggs A, Straume O, Akslen LA, Lifshits E, Byers LA, Xu L, Wu HK, Jänne P, Kobayashi S, Halmos B, Tenen D, Tang XM, Engelman J, Yeap B, Folkman J, Johnson BE and Heymach JV: Combined vascular endothelial growth factor receptor and epidermal growth factor receptor (EGFR) blockade inhibits tumor growth in xenograft models of EGFR inhibitor resistance. Clin Cancer Res 15(10): 3484-3494, 2009. PMID: 19447865. DOI: 10.1158/10780432.CCR-08-2904

7 Nakagawa K, Garon EB, Seto T, Nishio M, Aix SP, Paz-Ares L, Chiu CH, Park K, Novello S, Nadal E, Imamura F, Yoh K, Shih JY, Au KH, Moro-Sibilot D, Enatsu S, Zimmermann A, FrimodtMoller B, Visseren-Grul C, Reck M and RELAY Study Investigators: Ramucirumab plus erlotinib in patients with untreated, EGFR-mutated, advanced non-small-cell lung cancer (RELAY): A randomized, double-blind, placebo-controlled, phase 3 trial. Lancet Oncol 20(12): 1655-1669, 2019. PMID: 31591063. DOI: 10.1016/S1470-2045(19)30634-5

8 Watanabe M, Kawaguchi T, Isa S, Ando M, Tamiya A, Kubo A, Saka H, Takeo S, Adachi H, Tagawa T, Kakegawa S, Yamashita M, Kataoka K, Ichinose Y, Takeuchi Y, Sakamoto K, Matsumura A and Koh Y: Ultra-Sensitive Detection of the pretreatment EGFR T790M mutation in non-small cell lung cancer patients with an EGFR-activating mutation using droplet digital PCR. Clin Cancer Res 21(15): 3552-3560, 2015. PMID: 25882755. DOI: 10.1158/1078-0432.CCR-14-2151

9 Akamatsu H, Koh Y, Okamoto I, Fujimoto D, Bessho A, Azuma $\mathrm{K}$, Morita S, Yamamoto $\mathrm{N}$ and Nakagawa $\mathrm{K}$ : Clinical significance of monitoring EGFR mutation in plasma using multiplexed digital PCR in EGFR mutated patients treated with afatinib (West Japan Oncology Group 8114LTR study). Lung Cancer 131: 128-133, 2019. PMID: 31027689. DOI: 10.1016/ j.lungcan.2019.03.021

10 Saito H, Fukuhara T, Furuya N, Watanabe K, Sugawara S, Iwasawa S, Tsunezuka Y, Yamaguchi O, Okada M, Yoshimori K, Nakachi I, Gemma A, Azuma K, Kurimoto F, Tsubata Y, Fujita Y, Nagashima H, Asai G, Watanabe S, Miyazaki M, Hagiwara K, Nukiwa T, Morita S, Kobayashi K and Maemondo M: Erlotinib plus bevacizumab versus erlotinib alone in patients with EGFRpositive advanced non-squamous non-small-cell lung cancer (NEJ026): interim analysis of an open-label, randomised, multicentre, phase 3 trial. Lancet Oncol 20(5): 625-635, 2019. PMID: 30975627. DOI: 10.1016/S1470-2045(19)30035-X

11 Yu HA, Schoenfeld AJ, Makhnin A, Kim R, Rizvi H, Tsui D, Falcon C, Houck-Loomis B, Meng F, Yang JL, Tobi Y, Heller G, Ahn L, Hayes SA, Young RJ, Arcila ME, Berger M, Chaft JE, Ladanyi M, Riely GJ and Kris MG: Effect of osimertinib and bevacizumab on progression-free survival for patients with metastatic EGFR-mutant lung cancers: A phase 1/2 single-group open-label trial. JAMA Oncol 6(7): 1-8, 2020. PMID: 32463456. DOI: $10.1001 /$ jamaoncol.2020.1260

12 Yu HA, Paz-Ares L, Yang JC, Lee KH, Garrido P, Park K, Kim JH, Lee DH, Mao H, Wijayawardana SR, Gao L, Hozak RR, Chao BH and Planchard D: Phase 1 study of the efficacy and safety of ramucirumab in combination with osimertinib in advanced T790M-positive EGFR-mutant non-small cell lung 
cancer. Clin Cancer Res, 2020. PMID: 33046516. DOI: 10.1158/1078-0432.CCR-20-1690

13 Akamatsu H, Toi Y, Hayashi H, Fujimoto D, Tachihara M, Furuya N, Otani S, Shimizu J, Katakami N, Azuma K, Miura N, Nishino K, Hara S, Teraoka S, Morita S, Nakagawa K and Yamamoto N: Efficacy of osimertinib plus bevacizumab vs. osimertinib in patients with EGFR T790M mutation and nonsmall cell lung cancer previously treated with epidermal growth factor receptor-tyrosine kinase inhibitor. JAMA Oncol, 2020. DOI: $10.1001 /$ jamaoncol.2020.6758

14 Lin CC, Shih JY, Yu CJ, Ho CC, Liao WY, Lee JH, Tsai TH, Su KY, Hsieh MS, Chang YL, Bai YY, Huang DDR, Thress KS and Yang JCH: Outcomes in patients with non-small-cell lung cancer and acquired Thr790Met mutation treated with osimertinib: a genomic study. Lancet Respir Med 6(2): 107-116, 2018. PMID: 29249325. DOI: 10.1016/S2213-2600(17)30480-0

15 Tan K, Leong SM, Kee Z, Caramat PV, Teo J, Blanco MVM, Koay ESC, Cheong WK, Soh TIP, Yong WP and Pang A: Longitudinal monitoring reveals dynamic changes in circulating tumor cells (CTCs) and CTC-associated miRNAs in response to chemotherapy in metastatic colorectal cancer patients. Cancer Lett 423: 1-8, 2018. PMID: 29518480. DOI: 10.1016/j.canlet. 2018.02.039
16 Leonetti A, Sharma S, Minari R, Perego P, Giovannetti E and Tiseo M: Resistance mechanisms to osimertinib in EGFRmutated non-small cell lung cancer. Br J Cancer 121(9): 725737, 2019. PMID: 31564718. DOI: 10.1038/s41416-019-0573-8

17 Tone M, Inomata M, Awano N, Kuse N, Jo T, Yoshimura H, Minami J, Takeda K, Miyamoto S and Izumo T: Salvage chemotherapy following osimertinib in non-small cell lung cancer harboring epidermal growth factor receptor mutation. Anticancer Res 40(4): 2239-2246, 2020. PMID: 32234920. DOI: 10.21873/anticanres. 14186

Received December 10, 2020

Revised December 21, 2020

Accepted December 22, 2020 